\title{
Electron Polar Cap and the Boundary of Open Geomagnetic Field Lines
}

\author{
L. C. Evans ${ }^{1}$ and E. C. Stone ${ }^{2}$ \\ California Institute of Technology, Pasadena, California 91109
}

\begin{abstract}
A total of 333 observations of the boundary of the polar access region for electrons $\left(E_{e}>\right.$ $530 \mathrm{kev}$ ) provides a comprehensive map of the electron polar cap. The boundary of the electron polar cap, which should occur at the latitude separating open and closed field lines, is consistent with previously reported closed field line limits determined from trapped-particle data. The boundary, which is sharply defined, seems to occur at one of three discrete latitudes. Although the electron flux is generally uniform across the polar cap, a limited region of reduced access is observed about $10 \%$ of the time.
\end{abstract}

Although numerous observations of proton fluxes at low polar altitudes have been reported and discussed, observations of electron fluxes in the polar regions have been reported much less frequently [Vampola, 1967, 1969, 1971; McDiarmid and Burrows, 1970; West and Vampola, 1971; Maseley et al., 1971]. As was suggested by Vampola [1971] and Maseley et al. [1971], the low rigidity of $300-k e v$ electrons makes them effective small-scale probes of the geomagnetic field. Vampola used $\mathbf{2 5}$ observations of the electron polar cap and the lower-latitude 'quasi-trapped' regions to define the boundary of the electron polar cap and show that this boundary is also the boundary of the open field line region as mapped on to the polar cap. Vampola extrapolated his observations to quiet geomagnetic times by using observations of the trapping limit for $300-\mathrm{kev}$ electrons.

The observations reported here significantly extend these previous results. We present a total of 333 observations of the electron polar-cap boundary that were obtained from 211 satellite polar passes during geomagnetically quiet times and therefore require no extrapolation. These data represent a mapping of the boundary between open and closed geomagnetic field lines that is more comprehensive than that previously available by an order of magnitude. These data are shown to be consistent with previous electron polar-cap observations and with the trap-

${ }_{1}^{1}$ Now at NASA Ames Research Center, Moffett Field, California 94035.

${ }^{2}$ Alfred P. Sloan Research Fellow.

Copyright (C) 1972 by the American Geophysical Union. ping limit for $35-\mathrm{kev}$ electrons previously reported by McDiarmid and Burrows [1968].

These results were obtained from a joint University of Chicago and California Institute of Technology experiment on Ogo 4. The instrument, which was launched into a low-altitude polar orbit (apogee $910 \mathrm{~km}$, perigee $410 \mathrm{~km}$ ), contains two cosmic-ray detector systems. The primary detector system is a sandwich of absorbers and two circular Au-Si surface barrier detectors $\left(D_{1}\right.$ and $\left.D_{2}\right)$ inside a plastic scintillator anticoincidence cup $\left(D_{3}\right)$ that defines a vertical acceptance cone with a $30^{\circ}$ half-angle, resulting in a geometrical factor of $\sim 1.1 \mathrm{~cm}^{2}$ ster. Both Si detectors have nominal $240-\mu \mathrm{m}$ depletion depths, and the telescope is covered by a $2.6-\mathrm{mg} / \mathrm{cm}^{2}$ aluminized Mylar window. The energy loss in the front detector is pulse height analyzed for protons $\left(1.2 \leq E_{p} \leq 39 \mathrm{Mev}\right), \alpha$ particles $(4.4 \leq$ $\left.E_{\alpha} \leq 156 \mathrm{Mev}\right)$, and electrons $\left(0.45 \leq E_{\alpha} \lesssim 2\right.$ $\mathrm{Mev})$, Response of the rear detector $\left(D_{2}\right)$ indicates a more energetic event, i.e., $9.3 \leq E_{v} \leq 39$ $\mathrm{Mev}, 37 \leq E_{\alpha} \leq 156 \mathrm{Mev}$, or $0.7 \leq E_{\odot} \lesssim 3$ Mev. The counting rates $D_{1} \bar{D}_{3}, D_{2} \bar{D}_{3}, D_{1} D_{2} \bar{D}_{3}$, and $D_{3}$ are also monitored. The maximum effective geometrical factors for detecting electrons with $D_{1}$ and $D_{2}$ are $\sim 0.08 \mathrm{~cm}^{2}$ ster at 650 kev and $\sim 0.36 \mathrm{~cm}^{2}$ ster at $1.2 \mathrm{Mev}$, respectively; the efficiency for $D_{1} D_{2} \bar{D}_{3}$ electron events is $\left\langle 10^{-8}\right.$ [Lupton and Stone, 1972]. The anticoincidence cup $D_{3}$ is also sensitive to electrons above a threshold energy of $\sim 530 \mathrm{kev}$. The $D_{3}$ geometrical factor is $\sim 54 \mathrm{~cm}^{2}$ ster at threshold and increases to the omnidirectional value of $590 \mathrm{~cm}^{2}$ ster for penetrating particles. Since this telescope is mounted on the spacecraft so that the first 
three rates represent vertically incident (i.e., from the zenith) particles, it is referred to as the vertical telescope, and the monitored rates are referred to as $V_{1} \bar{V}_{3}, V_{2} \bar{V}_{3}, V_{1} V_{2} \bar{V}_{3}$, and $V_{3}$, respectively.

Although this instrument was not designed primarily to detect electrons, several periods of high electron fluxes have been observed in the polar-cap regions on board Ogo 4 . The contribution of electrons to the observed rates, which varies considerably, is typically $\lesssim 10 \%$ for the $V_{1} \bar{V}_{3}$ rate and $\lesssim 35 \%$ for the $V_{2} \bar{V}_{3}$ rate. Thus electrons normally constitute a rather minor constituent of the observed rates. There are periods, however, when the electron flux is sufficiently high in relation to the proton flux so that electrons become an identifiable constituent of the $V_{1} \bar{V}_{3}, V_{2} \bar{V}_{3}$, and $V_{3}$ rates. The identification of these periods and the identification of the rates as being predominantly due to electrons is illustrated in Figure 1. The $V_{2} \bar{V}_{3}$ and $V_{3}$ rates from this polar pass show a uniform electron polar-cap flux between $17 \mathrm{~h} 55 \mathrm{~m} 40 \mathrm{~s}$ UT and $18 \mathrm{~h} 04 \mathrm{~m} 40 \mathrm{~s}$ UT; beyond $18 \mathrm{~h} 05 \mathrm{~m} \mathrm{00s}$ UT the spacecraft had moved to invariant latitudes where the electrons did not have free access. That this enhancement can be associated strictly with electrons can be seen by comparing the $V_{2} \bar{V}_{3}$ rate (electrons and protons) with the $V_{1} V_{2} \bar{V}_{3}$ rate (protons only). To improve the

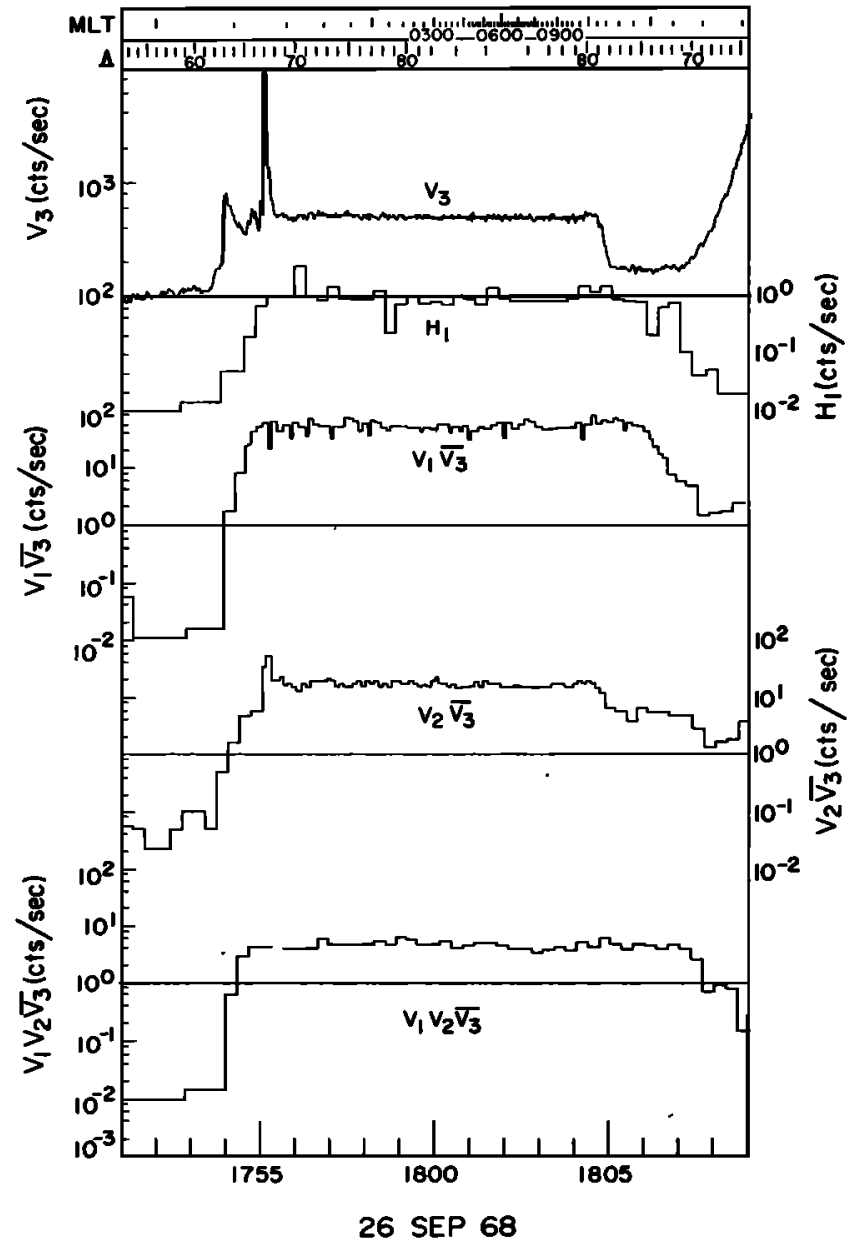

Fig. 1. Rates observed during a typical Ogo 4 polar pass $\left(|\Lambda| \geqslant 50^{\circ}\right)$, illustrating an enhanced electron polar-cap flux. These data are from the north polar pass of revolution 6291 on September 26, 1968. Invariant latitude $(\Lambda)$ and magnetic local time (MLT) are also indicated. 

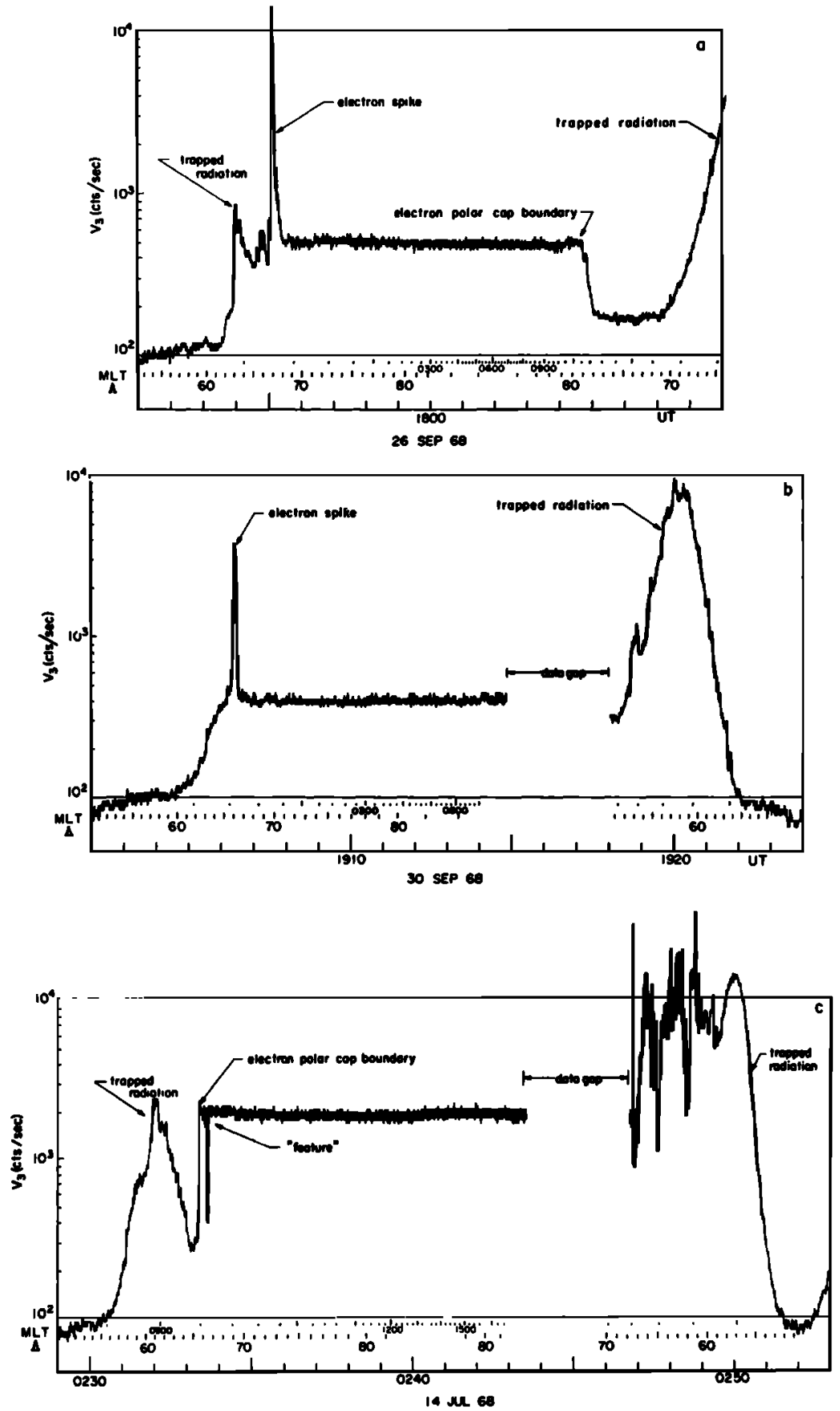

Fig. 2. High-resolution plots of the $V_{3}$ rate, showing characteristic electron polar-cap observations: ( $a$ ) $V_{3}$ rate shown in Figure 1. (b) An example of the type of electron spike associated with the edge of the electron polar cap between 2100 and 0200 MLT. (c) An example of a typical feature in the electron polar-cap flux. Invariant latitude $(\Lambda)$ and magnetic local time (MLT) are also indicated. Since Ogo 4 is in a low orbit, the trapped radiation intensity varies greatly depending on details of the polar pass. 
precision with which the electron polar-cap boundary could be specified, the $V_{3}$ rate was plotted on an expanded scale, as is shown in Figure 2a.

Figure $2 b$ illustrates the fact that the edge of the electron polar cap was normally associated with an electron spike near magnetic local midnight $(\sim 2100-0200 \mathrm{MLT})$. Figure $2 c$ is an example of an electron polar-cap observation showing a typical feature, i.e., the sharp depression at $02 \mathrm{~h} 33 \mathrm{~m} 40 \mathrm{~s}$ UT. These features appeared on 30 of the 257 polar passes, including 20 of 211 passes during geomagnetically quiet times. All the observed features are plotted in Figure 3.

A total of 333 observations of the edge of the electron polar cap were made during magnetically quiet periods, and the resultant map of the boundary determined from the $V_{3}$ rate is shown in Figure 3. Here the geomagnetic coordinates (invariant latitude and magnetic local time) of each observation of the boundary are indicated by a symbol. The apparent dependence on geomagnetic activity is not dis- cussed here. The values reported by Vampola [1971], which he extrapolated to magnetically quiet conditions, are shown for comparison. The excellent agreement between our direct measurement and Vampola's extrapolation confirms the validity of the extrapolation technique, which was based on the argument that at a given magnetic local time the invariant latitude difference between the stable trapping limit and the last closed field line was unaffected by latitude variations of the trapping limit.

Near magnetic local midnight $(\sim 2100-0200$ MLT) the boundary of the electron polar-cap region is normally associated with an electron spike, as is illustrated in Figure $2 a$ and $b$ and summarized in Figure 3. These spikes are identical to those identified previously as 'type 2' by Brown and Stone [1972], who interpreted these spikes as being associated with the last closed field lines. This association is consistent with the present interpretation that the spikes coincide with the edge of the open field line region.

There is a grouping of the data along three

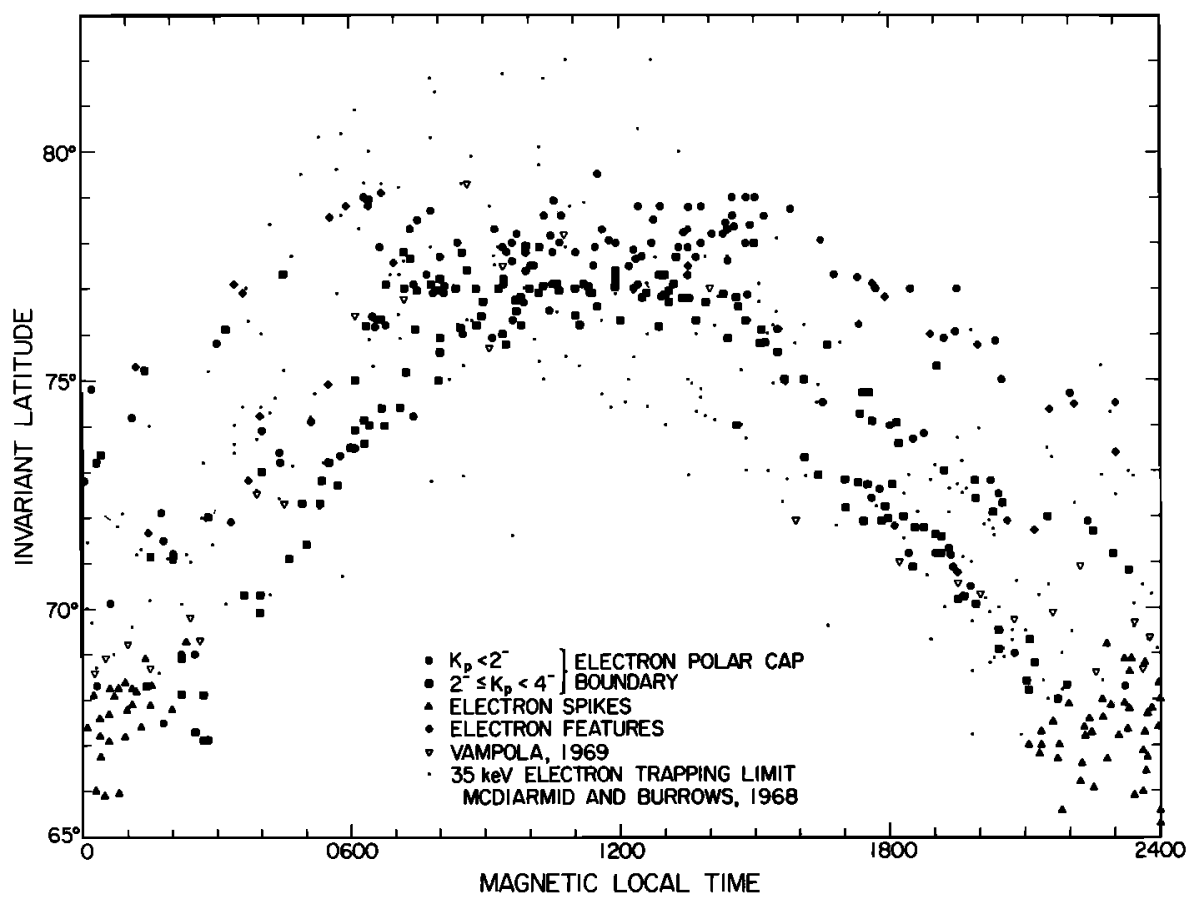

Fig. 3. The electron polar-cap boundary observed with $V_{s}\left(\boldsymbol{E}_{\boldsymbol{e}} \geq 530 \mathrm{kev}\right)$ in an invariant latitude and magnetic local time coordinate system, compared with similar observations by Vampola [1971] and with trapping-limit (background boundary) observations by McDiarmid and Burrows [1968]. Isolated regions of limited access (features) are also indicated. 
bands on either side of magnetic local noon; at $\sim 2000 \mathrm{MLT}$, for instance, these bands are near $\Lambda \approx 70^{\circ}, 72.5^{\circ}$, and $75.8^{\circ}$. These bands appear to merge near local noon and may be indistinguishable between 0800 and 1500 MLT. Numerous checks have been made to eliminate systematic biases, such as a longitude-dependent magnetic-field error. The first check proved that the north-polar and south-polar data independently exhibit the banding when plotted separately. As a second check, the data were sorted into $30^{\circ}$-wide geographic longitude bins. All bins contained numerous observations, and no bin contained events associated only with a single band.

As further evidence that the banding is not a systematic bias, reference is made to the extensive proton cutoff latitude observations obtained with the same instrument during many of the same solar-flare events as those of the electron polar-cap observations [Fanselow and Stone, 1972]. Although the cutoffs were determined to within $\pm 0.3^{\circ}$ (Figure $7 b$ of that paper), there were variations of $\pm 2^{\circ}$ during quiet times (their Figure 7a). However, there was no evidence of band structure with any of the field models used to organize the data [Fanselow and Stone, 1972, Figure 5], including the Jensen and Cain [1962] model used in this paper.

In addition to the checks for systematic biases, various physical correlations were investigated, the result being null. For example, no correlation was found that depended either on the interplanetary sector direction or on whether the interplanetary field had a north or a south component. However, correlation of the band structure with polar substorm activity has not yet been investigated.

Figure 3 also shows a comparison between the 530-kev electron polar-cap boundary and the high-latitude background boundary for 35 kev electrons previously reported by McDiarmid and Burrows [1968]. Both sets of data are interpreted as representing the boundary between 'open' and 'closed' geomagnetic-field lines. The agreement between the two sets of data is reasonable; the latitude spread of the $35-\mathrm{kev}$ data is consistent with the latitude separation of the three bands of 530-kev data, which may account for the large variation $\left( \pm 2^{\circ}\right)$ in the trapping limit position. Because of the relatively high spatial resolution of the electron polar boundary, additional studies may provide essential information on the dynamics of the magnetosphere.

Acknowledgments. We gratefully acknowledge the collaboration and support of Drs. J. A. Simpson and C. Y. Fan during various stages of the joint University of Chicago and California Institute of Technology program. The instrument was constructed by the Laboratory for Astrophysics and Space Research of the University of Chicago. D. H. Fairfield and N. F. Ness kindly provided the interplanetary magnetic-field data.

This work was performed under contract NAS 5-3095 and grant NGR 05-002-160. L. C. Evans received valuable support from the National Science Foundation and the State of California.

The Editor thanks J. R. Burrows and A. Vampola for their assistance in evaluating this report.

\section{ReFERENCES}

Brown, J. W., and E. C. Stone, High-energy electron spikes at high latitudes, J. Geophys. Res., 77, 3384, 1972.

Fanselow, J. L., and E. C. Stone, Geomagnetic cutoffs for cosmic-ray protons for seven energy intervals between 1.2 and $39 \mathrm{Mev}, J$. Geophys. Res., $77,3999,1972$.

Jensen, D. C., and J. C. Cain, An interim geomagnetic field (abstract), J. Geophys. Res., 67, 3568, 1962.

Lupton, J. E., and E. C. Stone, Electron scattering effects in typical cosmic ray telescopes, IEEE Trans. Nucl. Sci., NS-19, 562, 1972.

McDiarmid, I. B., and J. R. Burrows, Local time asymmetries in the high-latitude boundary of the outer radiation zone for the different electron energies, Can. J. Phys., 46, 49, 1968.

McDiarmid, I. B., and J. R. Burrows, Latitude profiles of low-energy solar electrons, $J$. Geophys. Res., 75, 3910, 1970.

Masley, A. J., W. P. Olson, and K. A. Pfitzer, Calculations of charged particle entry into the polar regions, Proc. Int. Conf. Cosmic Rays, $12 t h, 2,824,1971$.

Vampola, A. L., Energetic electrons at high latitudes after the September 2, 1966, solar flare (abstract), Eos Trans. AGU, 48, 169, 1967.

Vampola, A. L., Energetic electrons at latitudes above the outer-zone cutoff, J. Geophys. Res., $74,1254,1969$.

Vampola, A. L., Access of solar electrons to closed field lines, J. Geophys. Res., 76, 36, 1971.

West, H. I., Jr., and A. L. Vampola, Simultaneous observations of solar flare electron spectra in interplanetary space and within the earth's magnetosphere, Phys. Rev. Lett., $26,458,1971$.

(Received December 10, 1972; accepted June 28, 1972.) 